\title{
EXPERIMENTS WITH SINGLE TRAPPED YTTERBIUM IONS AT JPL
}

\section{Nan Yu and Lute Maleki}

Time and Frequency Sciences and Technology Group

Jet Propulsion Lab

California Institute of Technology

Pasadena, CA 91109

The work was carried out by the Jet Propulsion

Laboratory, California Institute of Technology, under a contract with the

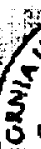
National Aeronautics and Space Administration.

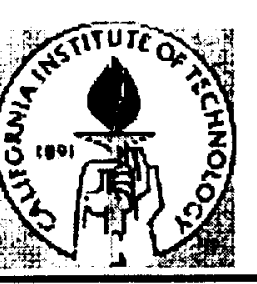




\section{$\underline{\text { Ytterbium ion level scheme }}$}

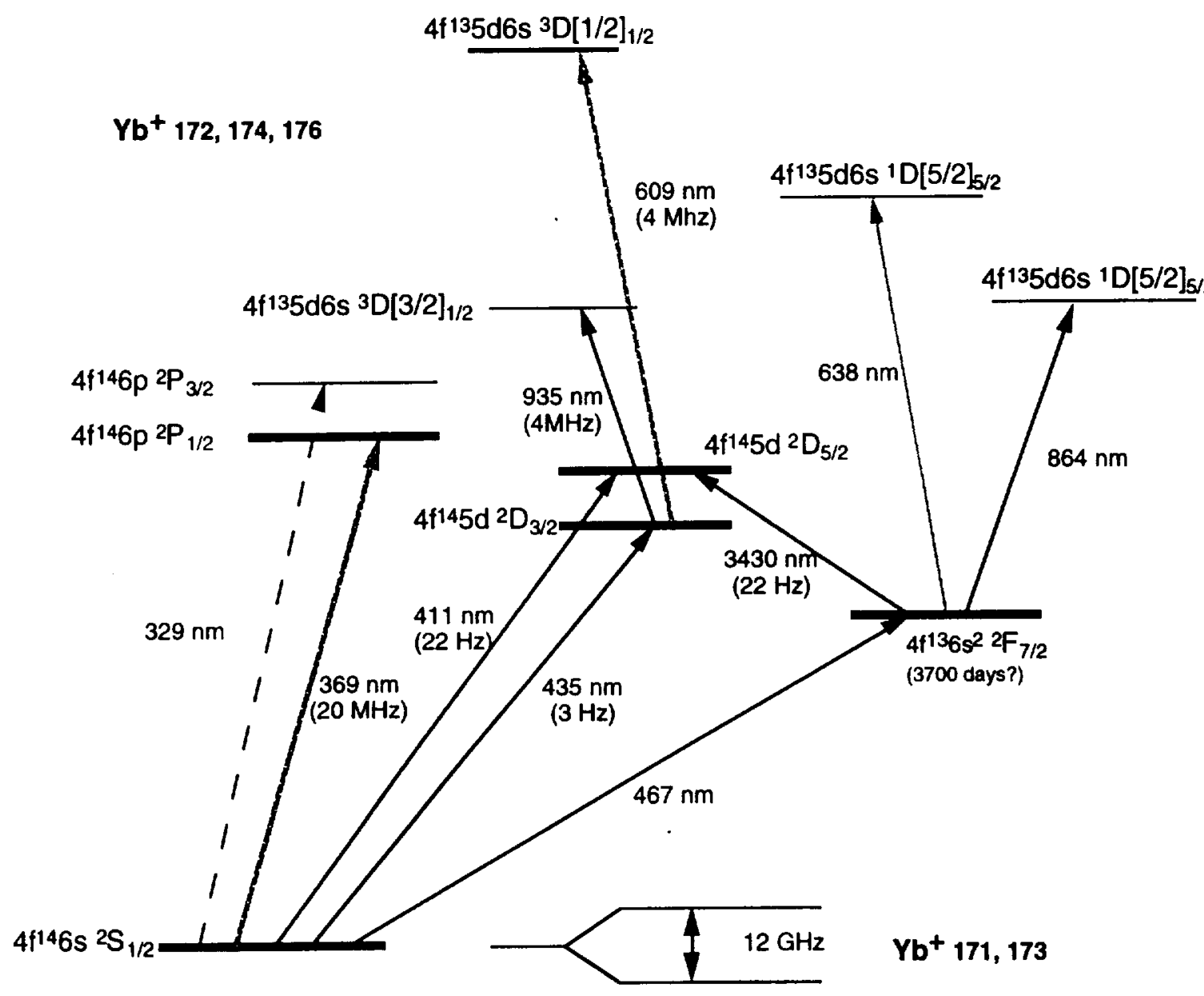

$369 \mathrm{~nm}$ cooling transition. $609 \mathrm{~nm} 935 \mathrm{~nm}, 638 \mathrm{~nm}$ clearing transitions.

$411 \mathrm{~nm}, 467 \mathrm{~nm}, 3.43 \mathrm{um}$ clock transitions.

$12 \mathrm{GHz}$ microwave trans. $329 \mathrm{~nm}$ auxiliary shelving transition. 


\section{Paul-Straubel rf trap and single ion image}

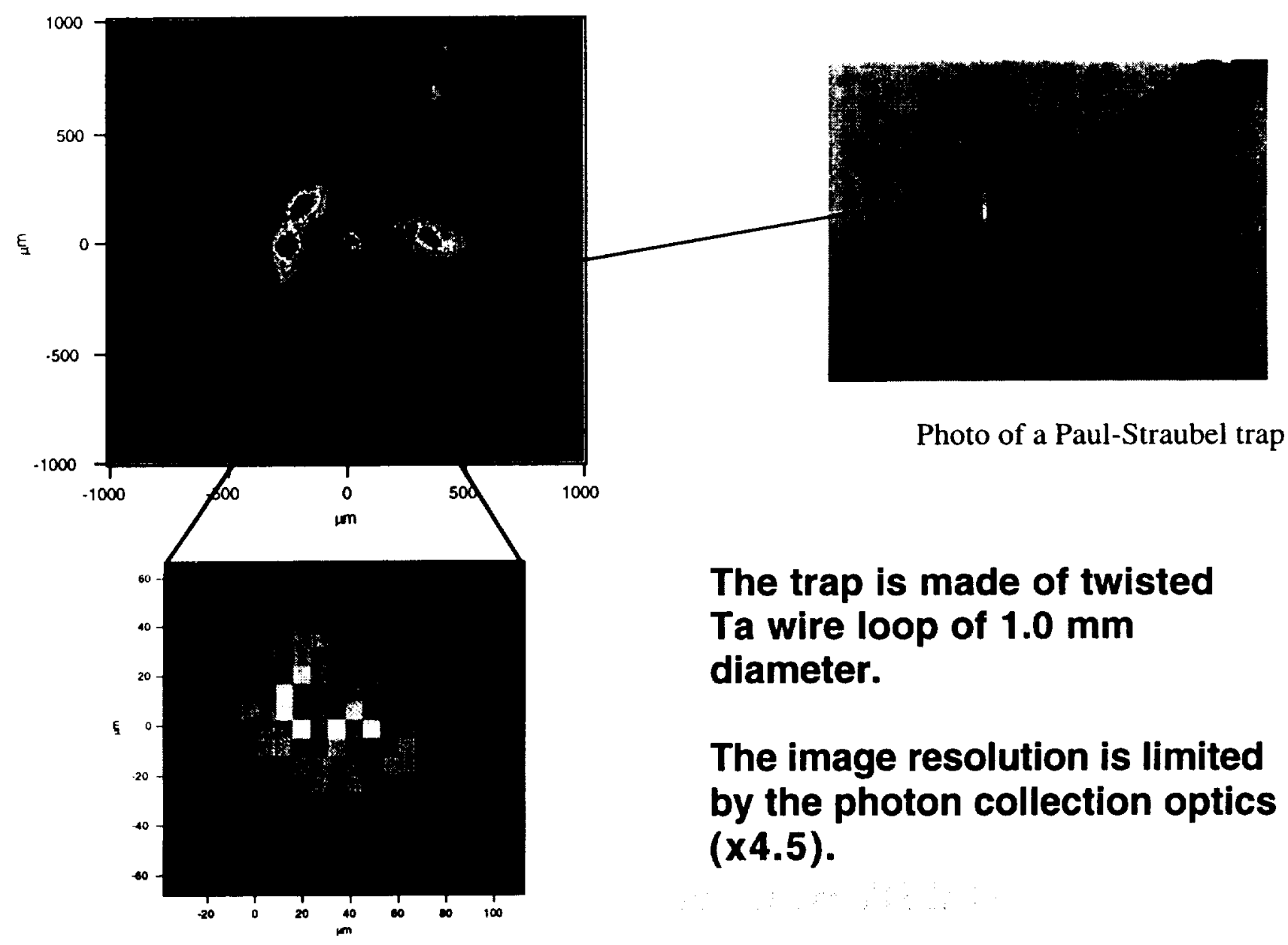

Single $\mathrm{Yb}+$ ion fluorescence image 


\section{D5/2 state lifetime measurement}
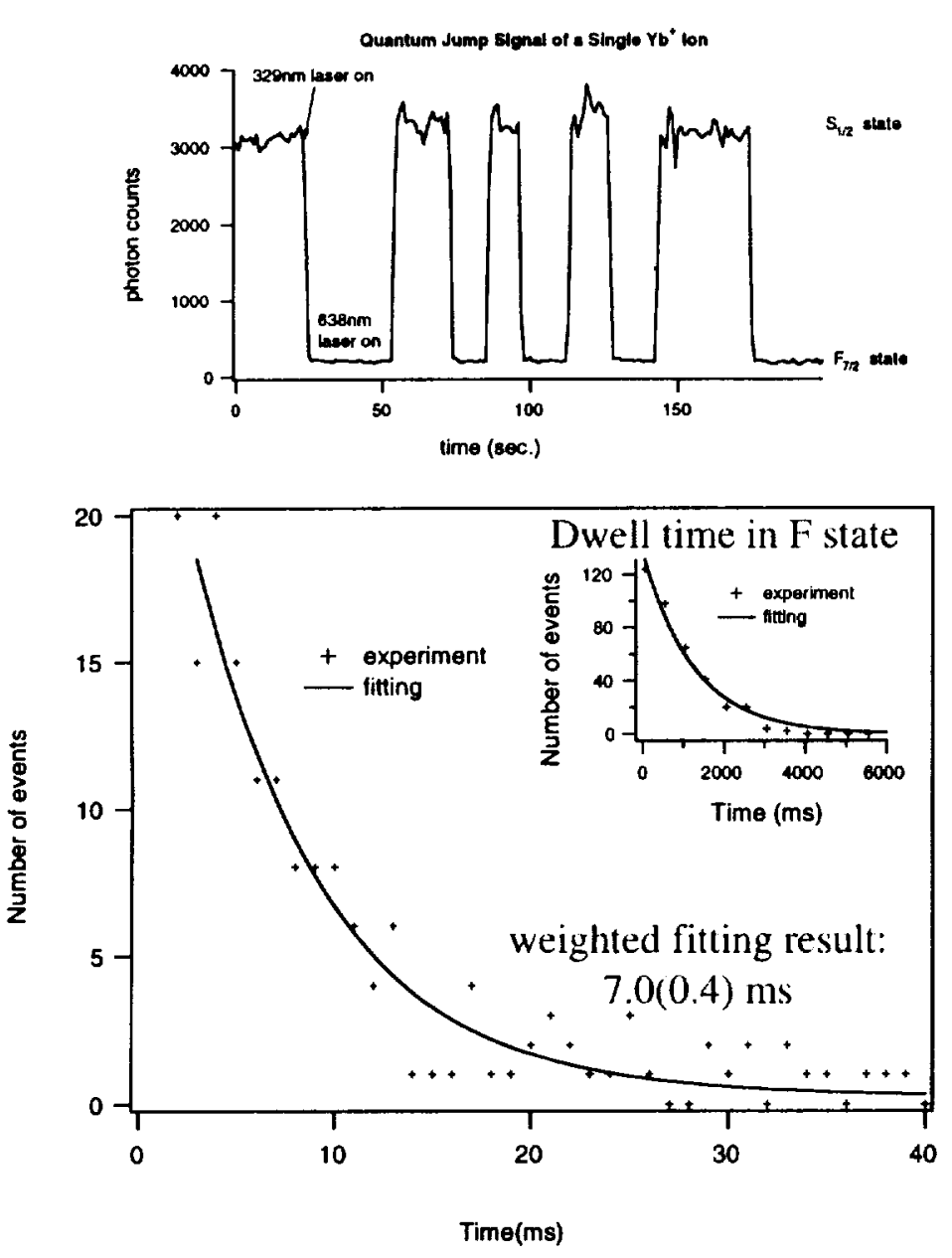

The lifetime of the $D_{5 / 2}$ state can be determined using the quantum jump technique. In this technique, continuous fluorescence photons are detected when the ion is in the ground state. An excitation of the ion into the $\mathrm{D}_{5 / 2}$ state will quench the : fluorescence completely until it decays spontaneously back to the ground state and the fluorescence resumes.

The on or off state of the fluorescence signal indicates whether the ion is in the $S_{1 / 2}$ or $D_{5 / 2}$ state. The average fluorescence offtime(dark period) gives the lifetime of the $\mathrm{D}_{5 / 2}$ state. 


\section{D3/2 state lifetime measurement}

\section{decay histograph}

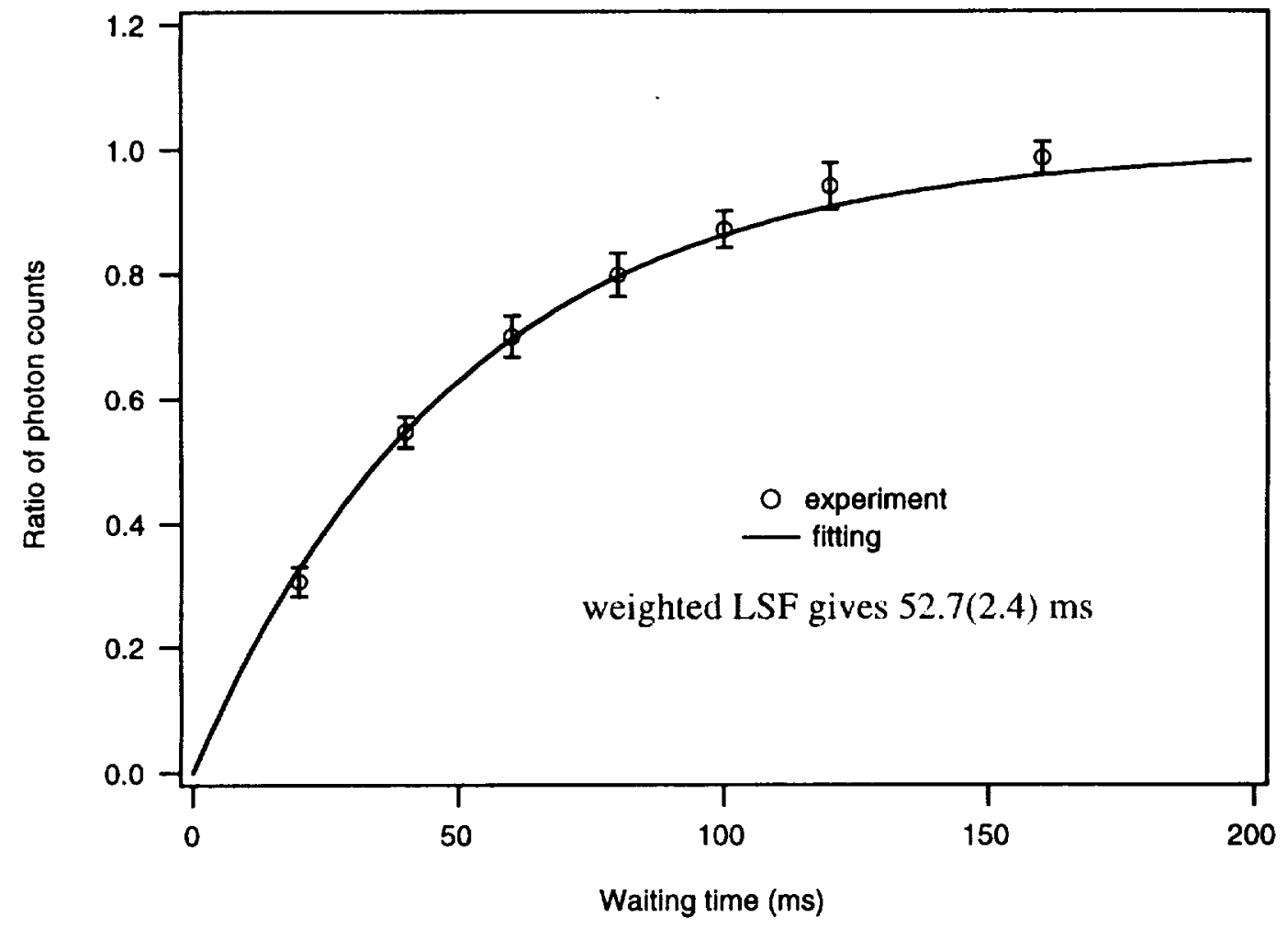

On average, 207 photons at $369 \mathrm{~nm}$ are scattered before the ion is repumped into $D_{3 / 2}$ state again, yielding the $\mathrm{P}_{1 / 2}$ branching ratio to be 0.0483 . 


\section{Trapped individual ions in an optical cavity}

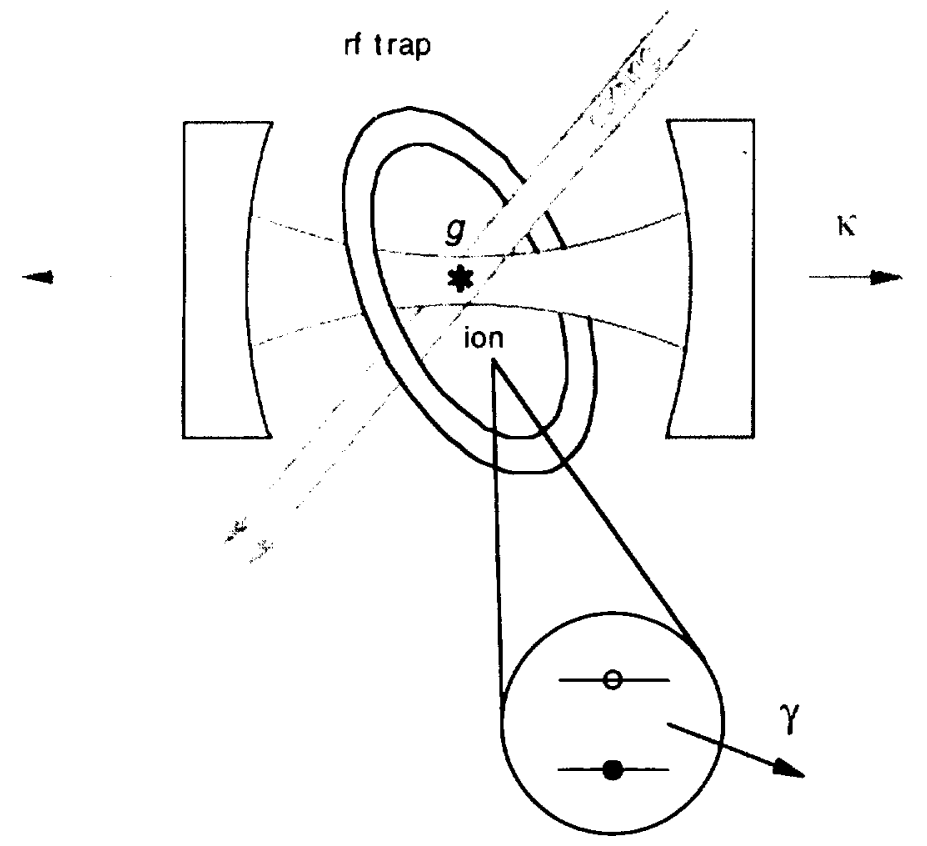

Interaction Hamiltonian:

$$
\begin{aligned}
& \hat{H}_{s}=\frac{\hbar \omega_{A}}{2} \hat{\sigma}^{z}+\hbar \omega_{c} \hat{a}^{\dagger} \hat{a}+i \hbar\left[g(\vec{r}) \hat{a}^{\dagger} \hat{\sigma}^{-}-g^{*}(\vec{r}) \hat{a} \hat{\sigma}^{+}\right] \\
& g(\vec{r})=\left(\frac{\mu^{2} \omega_{c}}{2 \hbar \epsilon_{0} V_{m}}\right)^{1 / 2} U(\vec{r})=g_{0} U(\vec{r})
\end{aligned}
$$

The strong coupling condition:

$g_{0}>(\gamma, \kappa)$

\section{Experimental challenges:}

* protecting mirror coating,

* avoid/circumvent dielectric charge-up,

* reducing cavity volume,

* 


\section{Initial exploratory system: experimental goals}

Feasibility demonstration:

- effects of atom beam collimation/contamination

- pulsed electron beam ion-loading/surface charge up problem

- ion translation capability, trap stability

- QED cavity locking/stabilization

- possible insitu surface discharge

Interesting physics to investigate:

- sw cooling

- cavity field mapping

- ion orbital size measurement

- laser transmission of occupied cavity

- QND atom state measurement through off resonance phase shift 
Future systems: trap-cavity integration II

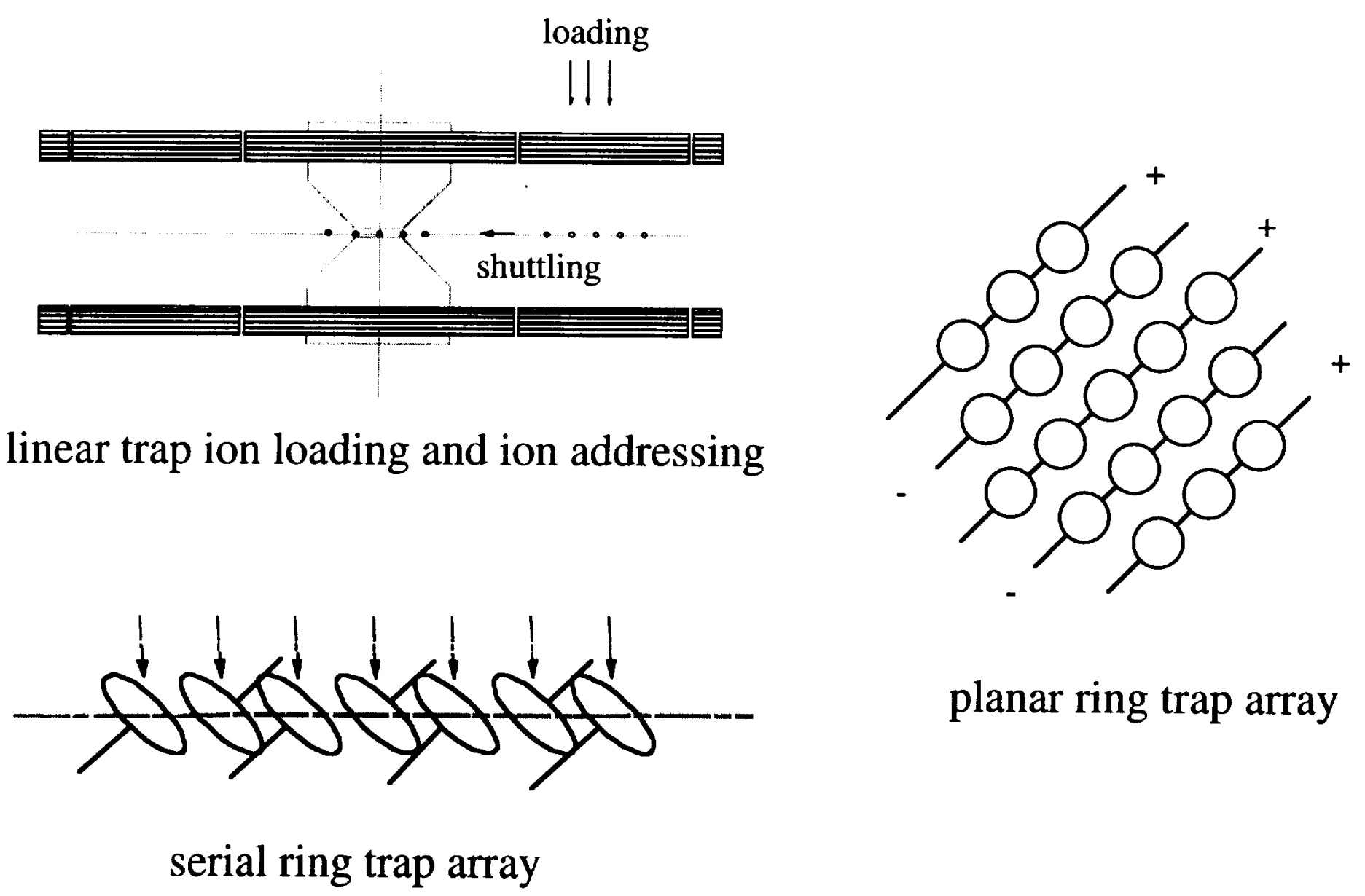

